\title{
Newly identified T cell subsets in mechanistic studies of food immunotherapy
}

\author{
Vanitha Sampath ${ }^{1}$ and Kari C. Nadeau ${ }^{1,2}$ \\ ${ }^{1}$ Sean N. Parker Center for Allergy and Asthma Research and ²Division of Pulmonary and Critical Care Medicine, Stanford University, Stanford, California, USA
}

\begin{abstract}
Allergen-specific immunotherapy has shown promise for the treatment of food allergy and is currently being evaluated in clinical trials. Although immunotherapy can induce desensitization, the mechanisms underlying this process are not completely understood. Recent advances in high-throughput technologies along with concomitant advances in data analytics have enabled monitoring of cells at the single-cell level and increased the research focus on upstream cellular factors involved in the efficacy of immunotherapy, particularly the role of T cells. As our appreciation of different T cell subsets and their plasticity increases, the initial simplistic view that restoring Th1/Th2 balance by decreasing Th2 or increasing Th1 responses can ameliorate food allergy is being enhanced by a more complex model involving other T cell subsets, particularly Tregs. In this Review, we focus on the current understanding of T cell functions in food allergy, tolerance, and immunotherapy.
\end{abstract}

\section{Introduction}

Nonallergic individuals maintain healthy immune unresponsiveness to commonly encountered food antigens via an active process; a breakdown or lack of development of this tolerogenic process underlies the pathophysiology of food allergy. The last few decades have seen significant increases in the prevalence of food allergies, which are currently estimated to affect $6 \%$ to $11 \%$ of the global population; the exact prevalence varies with geography, population studied, age, and methodology used (1-8). Rapid increases in the incidence of food allergy suggest that lifestyle and other environmental alterations, such as increased hygiene, consumption of processed foods, use of antibiotics, and exposure to environmental pollutants, likely influence its pathogenesis in those genetically predisposed to allergy (9).

Peanut is the most common food allergen in infants and children under 18 years in the United States, followed by milk, shellfish, tree nuts, egg, fin fish, wheat, and soy (9-11). Shellfish is the most common food allergy among US adults, followed by milk, peanut, tree nut, and fin fish (12). Allergies to more than one food are common, and approximately $40 \%$ of children with food allergy are estimated to be allergic to multiple foods (13). Childhood food allergies are also commonly associated with comorbid atopic conditions such as atopic dermatitis, asthma, and allergic rhinitis (14). Food allergy imposes a substantial burden on patients and their families due to dietary restrictions, increased anxiety, and social limitations (15-19).

There are currently no FDA-approved treatments for food allergy, and standard of care remains avoidance of allergenic

Conflict of interest: KCN has received funding from, is currently funded by, or cofounded the following: the NIH, Food Allergy Research \& Education, End Allergies Together, Before Brands, Alladapt Immunotherapeutics, Adare Pharmaceuticals, AstraZeneca, Novartis, Genentech, Astellas, DBV Technologies, ForTra, Aimmune Therapeutics, Regeneron, Nestle, Sanofi, ImmuneWorks, Cour, and the Environmental Protection Agency.

Copyright: (c) 2019 American Society for Clinical Investigation

Reference information: / Clin Invest. 2019;129(4):1431-1440.

https://doi.org/10.1172/JCl124605. foods and acute management of allergic reactions with antihistamines and epinephrine autoinjectors (20). However, accidental exposures are common (21), and even small doses can cause severe systemic reactions and even death; thus, safe and effective treatments for food allergy are an urgent and unmet need. The most promising treatment, allergen immunotherapy (AIT), is currently being evaluated in phase III clinical trials. In AIT, incrementally increasing doses of allergen are administered via various routes, such as oral, subcutaneous, sublingual, and epicutaneous (22); these different forms of AIT are discussed further in the section on immunotherapy. AIT has been shown to increase the allergen threshold dose (the amount of allergen that can be consumed without onset of allergic reactions). With immunotherapy, patients can potentially increase their ability to safely tolerate gradually increasing quantities of food allergens over a period of many months, eventually reaching a predetermined maintenance dose. At the end of the maintenance phase, increases in allergen threshold dose are assessed using oral food challenges. AIT's goal is to increase the ability to ingest foods at levels that, at a minimum, prevent risk of clinical reaction on accidental ingestion or, optimally, to levels that are consumed in normal diets. It is the only therapy known to alter the cellular and humoral immune response to allergens.

The immunologic mechanisms associated with immunotherapy are still not completely understood, and there are increased research efforts to further understand the mechanisms underlying food allergy and tolerance in order to develop safe and effective treatments. Natural tolerance is defined as a permanent state of clinical unresponsiveness to common innocuous foods. Current research indicates that clinical unresponsiveness achieved after successful AIT is not as durable as natural tolerance, as patients often become clinically resensitized after discontinuing regular allergen consumption. Continued ingestion of allergen is often required to maintain AIT's clinically unresponsive state, which is termed "desensitization" to distinguish it from the permanent unresponsive state of "tolerance." The clinical differences between 


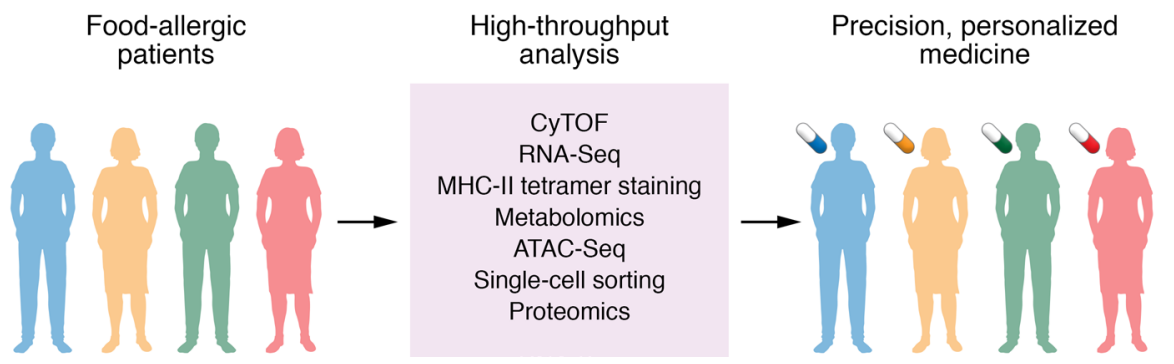

Figure 1. Systems biology, precision medicine, and personalized medicine. High-throughput systems biology technologies and concomitant advances in data analytics are enabling precision medicine and personalized care in food-allergic patients. ATAC-Seq, assay for transposase-accessible chromatin with high-throughput sequencing. desensitization with immunotherapy and natural tolerance suggest that there are mechanistic differences between these pathways.

Variations in desensitization's durability among patients are now being investigated to enable further understanding of the mechanistic differences between these variations and to enable more durable desensitization with AIT. As there are no available biomarkers to assess tolerance, and long-term data are limited, durability of desensitization is often determined by measures of sustained unresponsiveness (SU), defined as unresponsiveness to the allergen after a predetermined period of allergen discontinuation (generally a few weeks) following successful immunotherapy. SU rates from $27.5 \%$ to $50 \%$ were observed with peanut immunotherapy 4 to 6 weeks after discontinuation of peanut ingestion following successful desensitization (23-27), and preliminary data suggest that longer periods of immunotherapy treatment may increase SU durability. Additional long-term studies can help determine whether the observed rates of SU are indicative of tolerance.

In the past few decades, we have made great strides in elucidating some of the pathways involved in food allergy, natural tolerance, immunotherapy-induced desensitization, and SU. Initial research focused on the role of IgE antibodies in food allergy disorders. The identification of other downstream antibodies (IgG and IgA) and key cytokines (IL-4, IL-5, IL-9, IL-13, IL-10, IFN- $\gamma$, TGF- $\beta$, and others) has increased our understanding of humoral and cytokine responses in allergy. Current research is now focused on clarifying the role of upstream cellular factors ( $\mathrm{T}$ cells, B cells, type 2 innate lymphoid cells [ILC2s], antigen-presenting cells [APCs]) and humoral factors (IL-25, IL-33, thymic stromal lymphopoietin [TSLP]). In particular, T cells have garnered attention for their central role in mediating tolerance and food allergy as well as their potential as cell-based therapies (28-30). With the discovery of novel $\mathrm{T}$ cell subsets and recognition of $\mathrm{T}$ cell plasticity and $\mathrm{T}$ cell receptor (TCR) heterogeneity, the initial simplistic hypothesis that decreasing Th2 or increasing Th1 and restoring Th1/Th2 balance (31) would ameliorate food allergy is now being modified and refined as we gain further understanding of the complexities of $\mathrm{T}$ cell subsets. A number of $\mathrm{CD} 4^{+} \mathrm{T}$ cell subsets originating from naive T cells have now been identified (Th1, Th2, Th9, Th17, Th22, tissue resident memory [TRM] T cells, Tregs, $\gamma \delta$ T cells, follicular helper T [Tfh] cells, and invariant natural killer T [iNKT] cells), and their role in food allergy is an area of active research. In this Review, we focus on recent advances in our comprehension of $\mathrm{T}$ cell-mediated mechanisms of immunotherapy and its durability. We provide examples of human studies conducted in the last 3 years when available and also review studies using animal models of food allergy or other atopic diseases when relevant. We also dis- cuss the T cell-specific immune monitoring techniques that have made these discoveries possible.

\section{T cell immune monitoring in food allergy}

Skin prick and specific IgE tests, which are currently used to diagnose food allergy, determine reaction severity and prognosis, and monitor immune response to immunotherapy, are limited in reliability, sensitivity, and specificity. In recent years, a number of high-throughput technologies, in parallel with statistical and computational techniques, have facilitated systems biology approaches and furthered our ability to discern complex immune pathways, including those of T cells during AIT (32). The goal is to ultimately establish precision medicine that tailors treatments to each individual and provide personalized care for treating food allergy (Figure 1).

The ability to separate and analyze single cells as with FACS has increased our knowledge of immune cell populations $(33,34)$. Although advances in lasers, fluorochromes, and computer processing software have improved FACS analysis, it remains limited by the number of spectrally resolvable fluorochromes. Cytometry by time-of-flight mass spectrometry (CyTOF), a high-dimensional single-cell analytical technology, overcomes this limitation. CyTOF combines flow cytometry and mass spectrometry and can detect more than 40 intracellular and cell-surface targets in a single sampling of cells by labeling with metal isotypes rather than fluorochromes (35). It can identify all major leukocyte populations in human PBMCs from a single sample. A pilot study by Chinthrajah et al. evaluated cellular heterogeneity associated with participants with asthma alone, those with food allergy and asthma, and those with food allergy alone versus healthy controls and found that patients with food allergy (with and without asthma) had a lower percentage of memory $\mathrm{T}$ cells and Tregs compared with healthy controls. They also found significant differences in expression in Tregs, effector T cells, and central memory T cells, as well as altered ratios of Tregs to $\gamma \delta$ T cells, Th17 to $\gamma \delta$ T cells, and Th17 cells to Tregs between the different groups. Although larger studies are needed to confirm these associations, CyTOF can assist in identifying biomarkers and in the discovery of pathways and cell types that are dysregulated in food-allergic diseases (36). Coupling CyTOF with magnetic bead enrichment can detect cell types with low abundance. Using this combined technique, the frequency, phenotype, and function of antigen-specific T cells alone (based on their expression of CD154 [CD4OL] and CD69; ref. 37) were determined in greater detail than was previously possible with CyTOF alone (38). This technique appears very promising for shedding further light into the mechanisms underlying food allergy. 
Next-generation sequencing (NGS) technologies are revolutionizing our ability to characterize allergy at the genomic, transcriptomic (RNA-Seq), and epigenetic levels (e.g., methylation levels of each gene) (39). With NGS, millions of DNA fragments are attached to a solid surface or support and simultaneously sequenced in parallel. Using NGS, a study found differences in the fecal microbiome of food-allergic children, siblings, and healthy children that may offer clues to genetic and environmental factors that contribute to food allergy (40).

MHC class II (MHC-II) tetramer staining has emerged as an invaluable tool to $\mathrm{T}$ cell immunologists. It enables direct in vivo tracking of epitope-specific CD $4^{+} \mathrm{T}$ cells. MHC tetramers can bind up to four TCRs simultaneously, creating a much stronger interaction than single MHC:peptide complexes, which bind TCRs weakly. These tetramer complexes are now considered the gold standard for identifying allergen-specific T cells. Construction of these tetramers, however, requires prior knowledge of the specific peptide-MHC components involved, limiting its routine use (41-43). Further improvements to this technology include a next-generation peptide-MHC dodecamer, which is able to detect 2- to 5-fold more antigen-specific T cells than tetramers (44).

\section{T cells and the pathogenesis of food allergy}

$\mathrm{CD}^{+} \mathrm{T}$ cells are central mediators of food allergy. In individuals predisposed to allergic disease, $\mathrm{T}$ cells mediate allergic responses through release of Th2-type cytokines and IgE production. Pathogenic allergic responses by $\mathrm{T}$ cells can be mediated by increases in allergen permeability caused by disruption of the epithelium at barrier surfaces, such as the skin, gastrointestinal tract, or respiratory tract. Loss of epithelial barrier integrity can be caused by injury, inflammation, or genetic predisposition (e.g., the presence of lossof-function mutations in filaggrin, a key component of skin barrier function) (45). Figure 2 depicts the mechanism of food allergy sensitization via the gut. The main mechanisms underlying sensitization via the skin and lungs are similar to those of the gut, with immune deviations toward a Th2 profile and IgE production. Further details can be found in a number of excellent reviews (46-49).

In food allergy, disrupted epithelial cells produce the proinflammatory cytokines IL-25, IL-33, and TSLP, collectively called alarmins. Alarmins mediate their proinflammatory cascade primarily via APCs and naive T cells, but also via mucosal mast cells (50) and ILC2s (51). IL-33 upregulates OX4OL (the ligand for CD134) on DCs and drives differentiation of naive $\mathrm{CD} 4^{+} \mathrm{T}$ cells to Th2 cells (52). Th2 cells are the major cell type skewing immune reactions toward allergy by producing the cytokines IL-4, IL-5, IL-9, and IL-13. Mucosal mast cells and ILC2s also produce Th2type cytokines: ILC2s produce IL-5 and IL-13, and mucosal mast cells produce Th9 (52). Together, these type 2 cytokines promote IgE class switching by B cells and the binding of allergen-specific IgE antibodies to FceRI receptors on mast cells or basophils that primes the cells for allergic response (i.e., sensitization). When primed mast cells and basophils encounter an allergen, the FceRIbound IgE antibodies cross-link, activate degranulation, and release allergic mediators such as histamine, leukotrienes, and prostaglandins into the surrounding tissue. Symptoms of allergic response may include production of mucus, infiltration of eosinophils, vasodilation, smooth muscle contraction, and other effects.
In recent years, other T cells, such as Tfh and $\gamma \delta \mathrm{T}$, have been implicated in food allergy pathogenesis. TRM T cells are implicated in allergic asthma, and whether they play a role in food allergy is currently unclear (53). Most of these studies are in animal models, and further research to understand their role in humans is ongoing.

Tfh cells provide an alternate source of IL-4. Th2 and Tfh cells appear to have overlapping phenotypical and functional characteristics. In mice, airway exposure to peanut flour led to anaphylaxis and increased levels of cytokines (IL-4 and IL-21) and IgE via a Tfhdependent mechanism; genetic depletion of Tfh cells decreased IgE (54). Three subsets of Tfh cells (Tfh1, Tfh2, and Tfh17) have been identified. In patients with asthma, increases in the frequency of Tfh2 cells were observed, and Tfh2/Tfh1 ratios correlated with increases in $\operatorname{IgE}$, indicating a potential role for Tfh2 in allergy $(55,56)$.

$\gamma \delta \mathrm{T}$ cells are predominantly found in the intestinal epithelium and lamina propria and are therefore thought to contribute to immune surveillance and bridge innate and adaptive immunity. One study found that $\gamma \delta$ T cell activation leads to a breakdown of oral tolerance in mice (57). In another mouse study, induction of food allergy resulted in a decrease in the percentage of $\gamma \delta \mathrm{T}$ cells in intestinal tissues and Peyer's patches, but not in mesenteric lymph nodes or spleen. Blockade of the $\gamma \delta$ TCR resulted in elevated food allergic responses, characterized by increased IgE and Th2 cytokines, upon peanut sensitization (58). The majority of $\gamma \delta$ T cells are activated in an MHC-independent manner, in contrast to conventional MHC-restricted $\alpha \beta$ T cells. Human $\gamma \delta 2$ T cells were found to be a major source of IL-9, a cytokine known to mediate Th2-type allergic reaction (59). It is now understood that a number of $\gamma \delta \mathrm{T}$ cell subsets exist, and the role of each of these subtypes in allergic disease is being evaluated (60). Continuing research on $\gamma \delta \mathrm{T}$ cells will shed further light on their complexities.

\section{T cell mechanisms underlying tolerance}

Tolerance is an active process defined as a permanent state of unresponsiveness to commonly encountered antigens, even in the absence of regular allergen exposure. A large randomized controlled study of more than 600 infants from the Learning Early about Peanut Allergy (LEAP) study found that peanut tolerance was increased in infants who regularly consumed peanuts, indicating that consumption trained the immune system to become tolerant to peanuts in these children (61).

Tolerogenic responses primarily begin with the uptake of antigens at the intestinal epithelium. Allergen uptake and antigen presentation by DCs or macrophages to naive T cells direct their differentiation toward tolerogenic or allergenic subtypes. Initial studies suggested that this immune regulation involved homeostasis between T helper 1 (Th1) and T helper 2 (Th2) cells, with a Th2 response (IL-4, IL-5, and IL-13) indicative of an allergic response and a Th1-skewed response (IFN- $\gamma$ and TNF- $\alpha$ ) indicative of oral tolerance (31). This simplified theory is now being modified and refined as we gain further understanding of the complexities of $\mathrm{T}$ cell subsets.

Current research now indicates that Tregs play a key role in mediating tolerance (Figure 3). In tolerance, antigen-associated DCs migrate to the draining lymph nodes, where they induce differentiation of naive $\mathrm{T}$ cells into Tregs, which are then trans- 


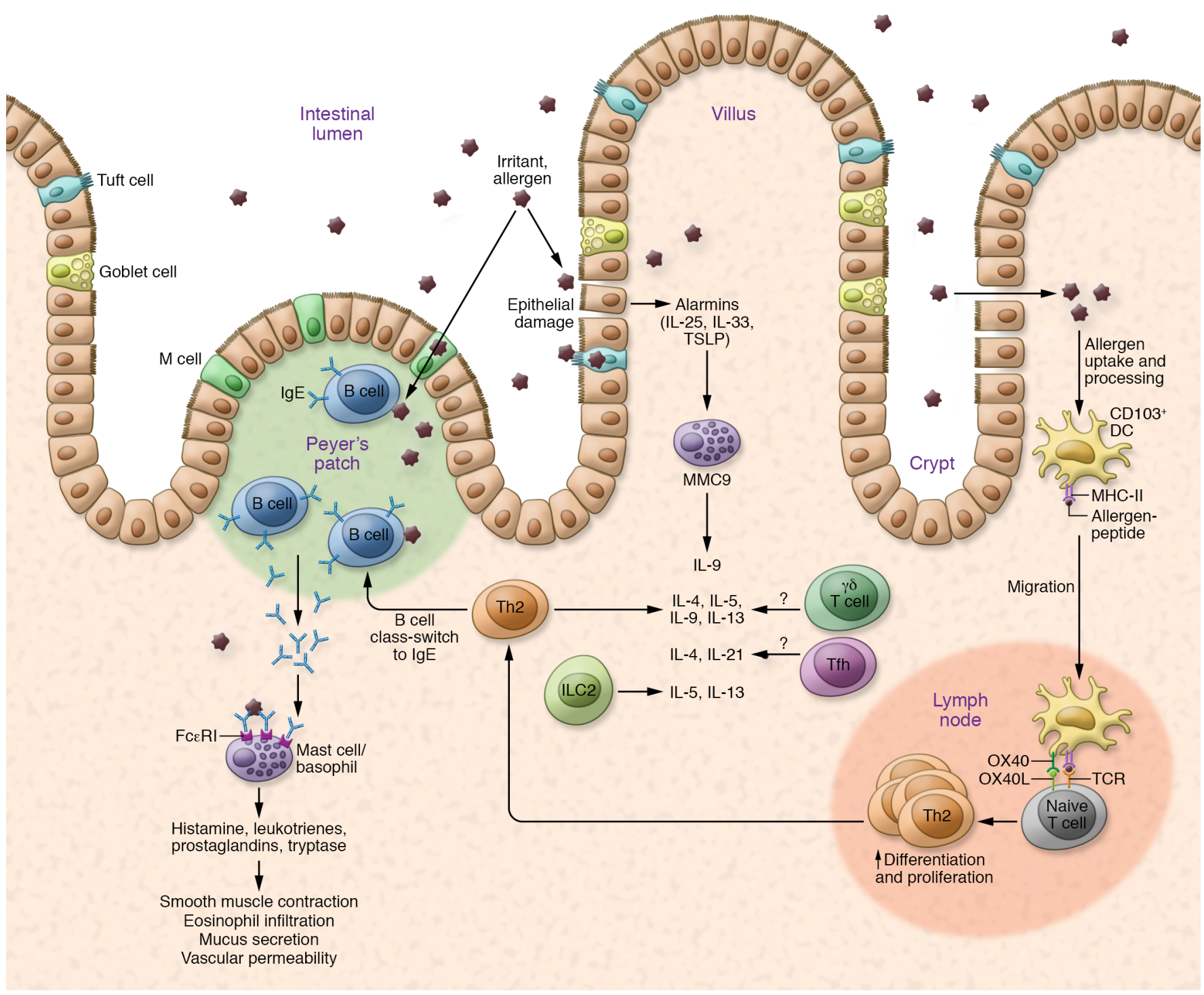

Figure 2. Mechanism of allergic reaction in food allergy. Allergic reaction is mediated by upregulation of Th2 cytokines and B cell class switching, which leads to production of IgE antibodies and degranulation of mast cells and basophils. Degranulation initiates allergic responses, such as smooth muscle contraction, eosinophil infiltration, mucus secretion, and vascular permeability. Other cells such as Tfh cells, $\gamma \delta$ T cells, and ILC2s are also thought to play a role in allergic reactions. Key cytokines involved are IL-25, IL-33, IL-4, IL-5, IL-13, and IL-9.

ported to the gut. This process is facilitated by TGF- $\beta$ and retinoic acid production and the expression of indoleamine 2,3-dioxygenase (IDO), C-C chemokine receptor type 9 (CCR9), and $\alpha_{4} \beta_{7}$ integrin. Several Treg subtypes have been associated with tolerance, including Foxp $3^{+}$Tregs, IL-10-secreting Foxp3 ${ }^{-}$Tregs (also known as Tr1 cells), and TGF- $\beta$-secreting T helper 3 (Th3) cells. Further details can be found in a number of excellent reviews on the topic $(46,48,62-68)$.

TCR diversity and multiplicity of effect of epitope repertoire diversity. Recent advances in high-throughput technologies have enabled our ability to measure TCR repertoire diversity and to further our understanding of the role of TCRs in tolerance. Analysis of proteomes based on the contact patterns of $\mathrm{T}$ cell-exposed motifs found extensive sharing of $\mathrm{T}$ cell epitope repertoires among the human proteome, gastrointestinal microbiome, and pathogenic bacteria, thus providing insights into the immune recognition and evasion of different classes of organisms and possibly offering clues for interventional strategies (69). A combination of multiplex PCR, Illumina sequencing, and IMGT/HighV-QUEST detected changes in TCR repertoire diversity following kidney transplantation that may eventually provide biomarkers to monitor immune status of tolerance (70). Based on studies in murine models, Fohse et al. concluded that an optimal and maximally broad organspecific Treg TCR repertoire is continuously shaped by inter- and intraclonal competition for diverse antigens. The study suggests that antigen diversity and high TCR diversity are crucial for optimal Treg expansion, peripheral reshaping of Treg TCR repertoire, and in vivo suppressive capacity (71). TCR databases with known antigenic specificities (such as VDJdb [https://vdjdb.cdr3.net] and GitHub [https://github.com/antigenomics/vdjdb-db]) are now enabling screening and analysis of repertoires for the presence and abundance of particular antigen-specific $\mathrm{T}$ cells. 


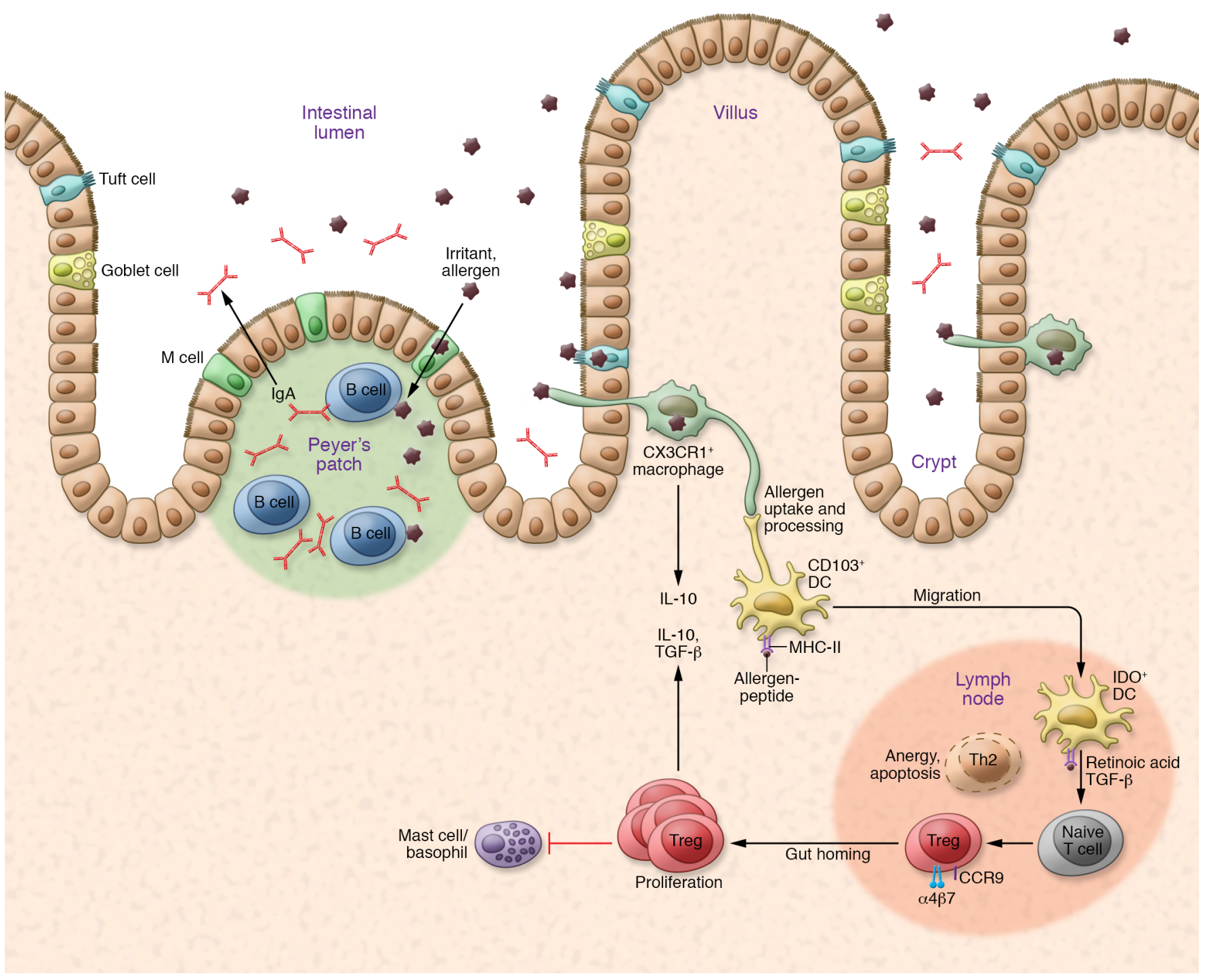

Figure 3. Mechanism of tolerance in food allergy in the gut. Tolerance is an active process: antigen-associated DCs migrate to the draining lymph nodes, where they induce differentiation of naive T cells into Tregs, which are then transported to the gut. This process is facilitated by TGF- $\beta$ and retinoic acid production and the expression of IDO and CCR9 and integrin $\alpha_{4} \beta_{7}$. Several subtypes of Tregs have been associated with tolerance, including Foxp $3^{+}$Tregs, IL-10-secreting Tr1 cells, and TGF- $\beta$-secreting Th3 cells.

\section{T cell mechanisms of food allergen immunotherapy}

AIT protocols currently comprise a number of routes of delivery and allergen doses. The most common forms of AIT for foods are oral immunotherapy (OIT) and sublingual immunotherapy (SLIT), which are currently in phase II/III clinical trials $(72,73)$. The skin's role as a major immunologic organ that may induce protection, sensitization, or tolerance is now recognized, and epicutaneous immunotherapy (EPIT) is showing promise in phase III clinical trials, although only a limited number of clinical trials using EPIT have been conducted to date (74). Subcutaneous immunotherapy (SCIT) is the predominant form of immunotherapy for aeroallergens, and initial clinical trials of SCIT for food allergies indicated that it was efficacious. However, because of unacceptably high rates of adverse reactions, SCIT was largely abandoned (75).

SLIT, OIT, and EPIT differ in allergen doses, efficacy, and safety. In EPIT, doses of allergens in the microgram range $(\sim 100-1000 \mu \mathrm{g}$ of protein) are delivered epicutaneously through a patch (76). In SLIT, doses are limited by the concentration of available extracts and the volume of liquid that can be held under the tongue. Thus, typical doses start with microgram levels of the allergenic protein and increase to milligram doses by maintenance. They are generally approximately 1000 times lower than OIT doses, which range from milligrams to grams (77). OIT's efficacy is high, with rates of clinical food allergy desensitization ranging from $80 \%$ to $90 \%$ (78-80) - much higher than those seen with SLIT and EPIT; however, OIT is also associated with a higher rate of systemic adverse events. OIT can be performed safely, but should be performed under clinical supervision so that adverse reactions can be treated promptly. With successful OIT and SLIT, patients can increase their allergenic threshold dose so that previously allergenic foods can be consumed at normal dietary amounts. Patients who successfully complete EPIT also have increases in allergenic threshold dose, but at lower levels, with the primary goal of reducing risk of accidental ingestion. 
$\mathrm{T}$ cell alterations that mediate allergic suppression after AIT are thought to be due to deviation of Th2-type responses to a Th1type response (immune deviation), induction of Tregs (immune regulation), deletion of Th2 cells (apoptosis), or Th2 cell anergy (unresponsiveness to antigen). It is currently unclear whether treatment strategies should be directed toward eliminating effector Th2 cells or toward increasing antigen-specific Treg responses. Identification of new subtypes of Th2 cells and Tregs has also revealed further complexities in the mechanism of food allergy.

The role of Tregs in desensitization has been observed by a number of studies; however, at present, there is no universal marker for identification of Tregs, and plasticity and overlapping function between Treg subtypes is likely (81). CD25 is currently used as a marker for Tregs, although it can be found on other cell types. Inducible Tregs, both Foxp $3^{+}$Tregs and Tr1 cells, have been shown to play key roles in desensitization during immunotherapy. Foxp $3^{+}$Tregs produce TGF- $\beta$ and inhibit Th 2 activity. Tr1 cells produce IL-10, which directly suppresses IgE, Th2-type cytokines, and associated proinflammatory cytokines (81). Patients successfully desensitized by peanut immunotherapy displayed Tregs with demethylation of Foxp3, but methylation increased again in patients who became resensitized to peanuts after a period of allergen discontinuation (25). Tolerogenic DCs were capable of inducing Tr1 cells at comparable levels in PBMCs derived from peanut-allergic and healthy controls; however, Tr1 cells induced in allergic patient PBMCs were functionally defective, as they were not anergic and had high Th2 cytokine production upon peanut-specific restimulation (82). Successful SLIT for peach allergy was linked to immunosuppression of allergen-specific effector $\mathrm{T}$ cells, potentially due to an increase of allergen-specific Tregs (83). Tregs mediate the shift in allergen-specific antibodies from the IgE to the IgG4 isotype and ameliorate IgE-mediated allergic reaction $(84,85)$. IgG4 antibodies may act as blocking antibodies, intercepting allergens before they cross-link mast cell FceRI-associated IgE. It has also been suggested that IgG4 costimulates the inhibitory IgG receptor Fc $\gamma$ RIIb, which can negatively regulate FceRI signaling and in turn inhibit effector cell activation (86).

Ryan et al. used MHC-II tetramers to identify allergen-specific $\mathrm{CD}^{+} \mathrm{T}$ cells and single-cell gene expression profiling and found that successful immunotherapy was associated with the development of an anergic Th2 phenotype, which was not present in either the pretreatment participants or healthy controls. No Treg induction was observed in any group (87). Using ex vivo MHC-II tetramer staining technology, a study by Wambre et al. further defined the role of Th2 cells by characterizing a population of memory Th2 cells (Th2a cells) found in peanut-allergic individuals. Further, these proinflammatory Th2a cells decreased in patients who benefited from peanut immunotherapy and could potentially be used as markers to determine prognosis with immunotherapy and for refining immunotherapy protocols (88). Single-cell profiling of peanut-responsive $\mathrm{T}$ cells obtained from patients with peanut allergy using flow cytometry and single-cell RNA sequencing revealed heterogeneous effector Th2 subsets but not Treg deficits, suggesting that anergy or deletion of Th2 cells is more likely in peanut allergy than upregulation of peanut-specific Tregs (89).

Alterations in $\mathrm{iNKT}$ and $\mathrm{CD} 8^{+} \mathrm{T}$ cells have also been observed with immunotherapy. iNKT cells can influence adaptive immune responses by producing vast amounts of cytokines. In children with cow's milk allergy, OIT induced a significant increase in peripheral blood iNKT cells, as well as induced their switch from a Th2 to a Th1 cytokine profile (90). In a mouse model, consumption of Food Allergy Herbal Formula-2, a medication based on traditional Chinese medicine, protected against peanut-induced anaphylaxis by elevating $\mathrm{CD}^{+} \mathrm{T}$ cell IFN- $\gamma$ production (91). In humans, $\mathrm{CD}^{+} \mathrm{T}$ cells expand in response to wheat ingestion in celiac disease (92). Our laboratory has shown that individuals with food challenge-proven, IgE-mediated peanut allergy have increases in allergen-specific $\mathrm{CD} 8^{+} \mathrm{T}$ cells, and that $\mathrm{CD} 8^{+} \mathrm{T}$ cells recognize specific peanut-derived peptides (93).

Based on our knowledge of $\mathrm{T}$ cells in food allergy, there has been tremendous progress in clinical research on next-generation treatments for food allergen immunotherapy. Vaccines such as $\mathrm{T}$ cell-based epitope vaccines and allergen-encoding DNA vaccines and adjunctive therapies such as omalizumab and probiotics are all currently in clinical trials for food allergy. These innovative therapies are described below.

\section{Novel vaccine immunotherapies and adjunctive treatments with immunotherapy}

Epitope-based synthetic peptide vaccines. With advancing knowledge of dominant $\mathrm{T}$ cell epitopes of major allergens, epitopespecific $\mathrm{T}$ cell responses are now being evaluated. Allergenic epitopes are considered safer than introducing native food allergens because they do not cross-link cell-bound IgE and thus do not trigger degranulation of mast cells and basophils. Epitope therapy requires identification of immunodominant $\mathrm{CD} 4^{+} \mathrm{T}$ cell epitopes of allergens and their ability to bind to HLA class II molecules. Use of peptide epitopes derived from allergens is a recently developed approach, and peptide vaccines are being tested in clinical trials $(94,95)$. In peanut-sensitized patients, differences in magnitude of $\mathrm{T}$ cell response, epitope specificity, and phenotype of symptomatic versus nonsymptomatic peanut-sensitized patients were observed. Reactivity against a pool of 19 immunodominant peptides (obtained from the major peanut allergens Ara h 1, 2, and 3) in symptomatic patients was dominated by IL-10, IL-17, and to a lesser extent IL-5. Tetramer staining in a very small subset of patients revealed higher levels of chemoattractant receptor-homologous molecule expressed on Th2 cells (CRTh2) expression and $\beta_{7}$ integrin expression in nonsymptomatic and symptomatic patients, respectively. CRTh2 is associated with allergic Th2 responses, and $\beta_{7}$ integrin is a gut-homing factor that plays a role in Treg regulation (96). A study investigated Ara h 1, 2, 3, 6, and 8 in eliciting T cell responses in peanut-allergic individuals and peanut-sensitized but not clinically reactive individuals. Using the CD154 upregulation assay and the class II tetramer technology, the study showed that Th2-type $\mathrm{T}$ cell responses in peanut-allergic individuals are directed against Ara h 1, 2, 3, and 6 and are dominated by an effector Th2 phenotype (97). Phase I clinical trials to assess the safety and tolerability of PVX108 (Aravax), a synthetic peptide representing $\mathrm{T}$ cell epitope sequences from major peanut allergens (Ara $\mathrm{h} 1$ and 2), are ongoing in peanut-allergic adults (Australian New Zealand Clinical Trials Registry ACTRN 12617000692336).

Allergen-encoding DNA vaccines to induce Th1 responses. Lysosomal-associated membrane protein 1 (LAMP-1) DNA plasmid 
vaccines are another novel immunotherapeutic approach for treating food allergies. The vaccines incorporate the sequence of $L A M P-1$ into plasmids encoding allergen. Upon immunization, APCs take up the plasmid and produce the allergen protein sequence as part of a fusion protein with LAMP-1. These fusion proteins are then directed into the APC lysosome and then the MHC-II pathway, stimulating the immune system via a CD $4^{+} \mathrm{Th}$ cell response. As the peptide allergen is not released from the APCs, the risk of allergic reaction to the vaccine is thought to be decreased (98). In a murine model, DNA-LAMP vaccines encoding the major allergens found in Japanese red cedar induced robust Th1-type immune responses (99). In clinical trials, the vaccine was found to be safe and effective (100). ASP0892, a DNA-LAMP peanut vaccine (Astellas Pharma Inc.), is designed to desensitize peanut-allergic individuals to Ara h 1,2 , and 3 via a Th1-mediated immune response. It is currently in clinical trials (ClinicalTrials. gov identifier NCT02851277).

\section{Adjunct biologics affecting T cells in immunotherapy}

Although immunotherapy has been shown to induce desensitization, its drawbacks include high rates of adverse reactions and the temporary nature of desensitization. Anti-IgE antibodies have been used as adjuvants in OIT to address these drawbacks. Omalizumab (Genentech/Novartis) is a monoclonal anti-IgE antibody that has been used as an adjunct to immunotherapy in a number of studies (101). It specifically binds to IgE molecules at the same epitope as FceRI, thereby reducing free IgE in circulation. Omalizum$\mathrm{ab}$ increases rate of allergen dose increases and decreases time to desensitization (102). In a study of patients with peanut allergy, OIT with adjunctive omalizumab promoted allergen desensitization through an initial omalizumab-dependent step that acutely depleted allergen-reactive $\mathrm{T}$ cells. The initial response was followed by an increase in allergen-specific activity of Tregs due to the reversal of their Th2 cell-like activity (103).

Other biologics that are being tested in clinical trials of food allergy are dupilumab (Sanofi and Regeneron Pharmaceuticals) and ANBO2O (AnaptysBio), which block proinflammatory cytokines that induce Th2-type immune responses. Dupilumab is an IL-4R $\alpha$ antibody that blocks IL-4 and IL-13 signaling, and ANB020 blocks IL-33 $(104,105)$.

\section{Microbiome, probiotics, prebiotics, and T cells in immunotherapy}

There is evidence that the microbiome plays an important role in the development of allergies (106). Epidemiologic studies and animal models of food allergy indicate that alterations in the microbiome due to modern diets can predispose to food allergy (107). Probiotics and prebiotics appear to play a role in allergic sensitization and disease by themselves or in combination with immunotherapy. Bacterial fermentation products, particularly short-chain fatty acids (SCFAs) such as acetate, propionate, and butyrate, are known to regulate T cells. SCFAs administered to mice either via drinking water, as an enema, or as a dietary precursor increased Foxp $3^{+}$Treg levels (108-110). In a mouse model of shrimp allergy, oral administration of the lactic acid bacterium Bacillus coagulans 09.712 resulted in strong induction of Foxp $3^{+}$
Tregs, production of IL-10, and amelioration of shrimp tropomyosin-induced allergic response via suppression of mTOR signaling (111). Supplementation with nondigestible plant-derived fructo-oligosaccharides (FOSs) improved OIT efficacy in a mouse model of cow's milk allergy. FOSs are known to directly modulate intestinal epithelial and immune cells in addition to acting as prebiotics (nutrients for microbiota). Increased levels of $\mathrm{Foxp}^{+}$Tregs and $\mathrm{LAP}^{+} \mathrm{Th} 3$ cells were observed in the mesenteric lymph nodes (112). In another mouse model of OVA-induced allergy, dietary intake of FOSs attenuated the induction of intestinal Th2 cytokines (113). In humans, preliminary evidence suggests that probiotics increase durability of desensitization. Coadministration of a probiotic (Lactobacillus rhamnosus CGMCC 1.3724) with peanut OIT in a randomized, double-blind, placebo-controlled trial was more effective at inducing SU than cotreatment with placebo $(114,115)$; however, the mechanisms by which they mediate their effects need to be further explored. In a study of shellfish allergy, oral administration of the probiotic strain Bifidobacterium infantis 14.518 was found to alter gut microbiota composition, induce Treg differentiation, and suppress Th2-biased response (116). In children with primary sclerosing cholangitis and inflammatory bowel disease, treatment with oral vancomycin, an antibiotic generally used to treat Clostridium difficile-associated diarrhea, increased peripheral levels of Foxp3 ${ }^{+}$Tregs (117). Mice deficient in inducible Tregs had altered gut microbiota and spontaneously developed hallmarks of allergic inflammation and asthma, suggesting that inducible Tregs have a function in ameliorating allergic-type inflammation at mucosal interfaces (118).

\section{Conclusions}

Food allergy is a complex and heterogeneous disease. Food allergy differs in offending allergen type, epitope, clinical presentation, and severity of reaction. The parallel advances of high-throughput technologies and big data analytics that are now available hold promise for precision medicine and personalized care by further elucidating the complex pathways that interact in mediating food allergy. T cells play a pivotal role in mediating response to allergens, and these technologies are assisting with further classification of subtypes and function. With the use of CyTOF, NGS, and the development of novel reagents for MHC class II tetramer staining, we have made tremendous progress in the last few decades in expanding our knowledge of mechanisms underlying food allergy, tolerance, and desensitization with immunotherapy. The knowledge gleaned from mechanistic studies using mouse models of food allergy are now being translated into clinical trials. We are also learning from animal studies and clinical trials on other atopic diseases such as allergic asthma, atopic dermatitis, and others. Current challenges include the limited availability of allergen-specific tetramer reagents, the limited number of antibodies for surface markers relevant to allergy research, the low frequency of food allergen-specific $T$ cells, and the need for prior knowledge of HLA types in patients. Immunodominant peptides are currently available mainly for peanuts and milk, and research into immunodominant peptides for other allergens is under way.

In humans, most studies of food allergy have used PBMCs rather than relevant gastrointestinal tissues, and this is a major 
shortcoming. With the use of a novel technique, multiplexed ion beam imaging (MIBI), and tissues obtained from gut biopsies, we hope that this shortcoming can be addressed in the future. With MIBI, antibodies are labeled with metallic elements, and tissues can be scanned using an ion beam to reveal more than 100 proteins simultaneously in a single cell. In addition to measuring protein levels on individual cells, it also provides information about cell morphology and localization. This novel technique has been used to analyze paraffin-embedded human breast cancer sections (119) and holds promise for use in food allergy research in identifying distinctive proteins in allergic and healthy individuals and assisting with targeted therapies and clinical diagnostics.

The future of food allergy research looks bright. Research on biologics and probiotics as adjunctive therapies with immunotherapy and novel vaccines for food allergy are under way. It is likely that approved treatments for food allergy will be a reality within the next 5 to 10 years.

\section{Acknowledgments}

We thank the NIH (grants U19AI104209, R01AI140134-01, U19AI070535, and U01AI140498-01), the Sean N. Parker Center for Allergy and Asthma Research at Stanford University, Food Allergy Research \& Education (FARE), the Myra Reinhard Foundation, End Allergies Together (EAT), the Hartman Family Foundation, the Naddisy Foundation, and Jeff and MacKenzie Bezos for their generous support.

Address correspondence to: Kari C. Nadeau, Sean N. Parker Center for Allergy and Asthma Research at Stanford University, 269 Campus Drive, CCSR 3215, MC 5366, Stanford, California 943055101, USA. Phone: 650.498.6073; Email: knadeau@stanford.edu.
1. Jackson KD, Howie LD, Akinbami LJ. Trends in allergic conditions among children: 453 United States, 1997-2011. NCHS Data Brief. 2013;(121):1-8

2. Nwaru BI, et al. Prevalence of common food allergies in Europe: a systematic review and meta-analysis. Allergy. 2014;69(8):992-1007.

3. Osborne NJ, et al. Prevalence of challengeproven IgE-mediated food allergy using population-based sampling and predetermined challenge criteria in infants. J Allergy Clin Immunol. 2011;127(3):668-676.e1.

4. Rinaldi M, Harnack L, Oberg C, Schreiner P, St Sauver J, Travis LL. Peanut allergy diagnoses among children residing in Olmsted County, Minnesota. J Allergy Clin Immunol. 2012;130(4):945-950.

5. Sicherer SH, Muñoz-Furlong A, Godbold JH, Sampson HA. US prevalence of selfreported peanut, tree nut, and sesame allergy: 11-year follow-up. JAllergy Clin Immunol. 2010;125(6):1322-1326.

6. Soller L, et al. Overall prevalence of self-reported food allergy in Canada. J Allergy Clin Immunol. 2012;130(4):986-988.

7. Gupta RS, et al. Prevalence and severity of food allergies among US adults. JAMA Netw Open. 2019;2(1):e185630.

8. Loh W, Tang MLK. The epidemiology of food allergy in the global context. Int J Environ Res Public Health. 2018;15(9):E2043.

9. Sicherer SH, Sampson HA. Food allergy: epidemiology, pathogenesis, diagnosis, and treatment. JAllergy Clin Immunol. 2014;133(2):291-307.

10. Gupta RS, et al. The public health impact of parent-reported childhood food allergies in the United States. Pediatrics. 2018;142(6):e20181235.

11. Grief SN. Food allergies. Prim Care. 2016;43(3):375-391.

12. Gupta RS, et al. Prevalence and severity of food allergies among US adults. JAMA Netw Open. 2019;2(1):e185630.

13. Gupta RS, et al. The prevalence, severity, and distribution of childhood food allergy in the United States. Pediatrics. 2011;128(1):e9-e17.

14. Spergel JM. From atopic dermatitis to asthma: the atopic march. Ann Allergy Asthma Immunol.
2010;105(2):99-106.

15. Primeau MN, et al. The psychological burden of peanut allergy as perceived by adults with peanut allergy and the parents of peanut-allergic children. Clin Exp Allergy. 2000;30(8):1135-1143.

16. Springston EE, Smith B, Shulruff J, Pongracic J, Holl J, Gupta RS. Variations in quality of life among caregivers of food allergic children. Ann Allergy Asthma Immunol. 2010;105(4):287-294.

17. Avery NJ, King RM, Knight S, Hourihane JO. Assessment of quality of life in children with peanut allergy. Pediatr Allergy Immunol. 2003;14(5):378-382.

18. Branum AM, Lukacs SL. Food allergy among children in the United States. Pediatrics. 2009;124(6):1549-1555.

19. Gupta RS, Kim JS, Barnathan JA, Amsden LB, Tummala LS, Holl JL. Food allergy knowledge, attitudes and beliefs: focus groups of parents, physicians and the general public. BMC Pediatr. 2008;8:36.

20. Boyce JA, et al. Guidelines for the diagnosis and management of food allergy in the United States: report of the NIAID-sponsored expert panel. JAllergy Clin Immunol. 2010;126(6 suppl):S1-S58.

21. Vander Leek TK, Liu AH, Stefanski K, Blacker B, Bock SA. The natural history of peanut allergy in young children and its association with serum peanut-specific IgE. J Pediatr. 2000;137(6):749-755.

22. Kostadinova AI, Willemsen LE, Knippels LM, Garssen J. Immunotherapy - risk/benefit in food allergy. Pediatr Allergy Immunol. 2013;24(7):633-644

23. Burks AW, et al. Oral immunotherapy for treatment of egg allergy in children. $N$ Engl J Med. 2012;367(3):233-243.

24. Jones SM, et al. Long-term treatment with egg oral immunotherapy enhances sustained unresponsiveness that persists after cessation of therapy. JAllergy Clin Immunol. 2016;137(4):1117-1127.e10.

25 . Syed A, et al. Peanut oral immunotherapy results in increased antigen-induced regulatory T-cell function and hypomethylation of forkhead box protein 3 (FOXP3). J Allergy Clin Immunol. 2014;133(2):500-510.

26. Wood RA, et al. A randomized, double-blind, placebo-controlled study of omalizumab combined with oral immunotherapy for the treatment of cow's milk allergy. J Allergy Clin Immunol. 2016;137(4):1103-1110.e11.

27. Vickery BP, et al. Sustained unresponsiveness to peanut in subjects who have completed peanut oral immunotherapy. J Allergy Clin Immunol. 2014;133(2):468-475.

28. Baranyi U, Gattringer M, Valenta R, Wekerle T. Cell-based therapy in allergy. Curr Top Microbiol Immunol. 2011;352:161-179.

29. Roybal KT, Lim WA. Synthetic immunology: hacking immune cells to expand their therapeutic capabilities. Annu Rev Immunol. 2017;35:229-253.

30. Saidova A, Hershkop AM, Ponce M, Eiwegger $\mathrm{T}$. Allergen-specific $\mathrm{T}$ cells in IgE-mediated food Allergy. Arch Immunol Ther Exp (Warsz). 2018;66(3):161-170.

31. Turcanu V, Maleki SJ, Lack G. Characterization of lymphocyte responses to peanuts in normal children, peanut-allergic children, and allergic children who acquired tolerance to peanuts. J Clin Invest. 2003;111(7):1065-1072.

32. Dhondalay GK, et al. Food allergy and omics. JAllergy Clin Immunol. 2018;141(1):20-29.

33. Cossarizza A, et al. Guidelines for the use of flow cytometry and cell sorting in immunological studies. Eur J Immunol. 2017;47(10):1584-1797.

34. Kunicki MA, Amaya Hernandez LC, Davis KL, Bacchetta R, Roncarolo MG. Identity and diversity of human peripheral Th and $\mathrm{T}$ regulatory cells defined by single-cell mass cytometry. J Immunol. 2018;200(1):336-346.

35. Leipold MD, Maecker HT. Phenotyping of live human PBMC using CyTOF ${ }^{\mathrm{TM}}$ mass cytometry. Bio Protoc. 2015;5(2):e1382.

36. Chinthrajah RS, et al. High dimensional immune biomarkers demonstrate differences in phenotypes and endotypes in food allergy and asthma. Ann Allergy Asthma Immunol. 2018;121(1):117-119.e1.

37. Rust BJ, Wambre E. Human immune monitoring techniques during food allergen immunotherapy. Curr Allergy Asthma Rep. 2017;17(4):22.

38. Lin D, Maecker HT. Mass cytometry assays for antigen-specific T cells using CyTOF. Methods Mol Biol. 2018;1678:37-47.

39. Yohe S, Thyagarajan B. Review of clinical next-generation sequencing. Arch Pathol Lab Med. 2017;141(11):1544-1557. 
40. Kourosh A, et al. Fecal microbiome signatures are different in food-allergic children compared to siblings and healthy children. Pediatr Allergy Immunol. 2018;29(5):545-554.

41. Newell EW, Sigal N, Nair N, Kidd BA, Greenberg HB, Davis MM. Combinatorial tetramer staining and mass cytometry analysis facilitate T-cell epitope mapping and characterization. Nat Biotechnol. 2013;31(7):623-629.

42. Van Hemelen D, et al. HLA class II peptide tetramers vs allergen-induced proliferation for identification of allergen-specific $\mathrm{CD} 4 \mathrm{~T}$ cells. Allergy. 2015;70(1):49-58.

43. Archila LL, Kwok WW. Tetramer-guided epitope mapping: a rapid approach to identify HLArestricted T-cell epitopes from composite allergens. Methods Mol Biol. 2017;1592:199-209.

44. Huang J, et al. Detection, phenotyping, and quantification of antigen-specific $\mathrm{T}$ cells using a peptide-MHC dodecamer. Proc Natl Acad Sci U S A. 2016;113(13):E1890-E1897.

45. Venkataraman D, et al. Filaggrin loss-of-function mutations are associated with food allergy in childhood and adolescence. J Allergy Clin Immunol. 2014;134(4):876-882.e4.

46. Chinthrajah RS, Hernandez JD, Boyd SD, Galli SJ, Nadeau KC. Molecular and cellular mechanisms of food allergy and food tolerance. J Allergy Clin Immunol. 2016;137(4):984-997.

47. Berin MC. Pathogenesis of IgE-mediated food allergy. Clin Exp Allergy. 2015;45(10):1483-1496.

48. Sicherer SH, Sampson HA. Food allergy: a review and update on epidemiology, pathogenesis, diagnosis, prevention, and management. J Allergy Clin Immunol. 2018;141(1):41-58.

49. Lozano-Ojalvo D, Berin C, Tordesillas L. Immune basis of food allergic reactions to food [published online ahead of print November 20, 2018]. J Investig Allergol Clin Immunol. https://doi. org/10.18176/jiaci.0355.

50. Barrett NA, Austen KF. Licensed to ill: IL-9 generation in immature mast cells permits food-elicited anaphylaxis. Immunity. 2015;43(4):626-628.

51. Bernink JH, Germar K, Spits H. The role of ILC2 in pathology of type 2 inflammatory diseases. Curr Opin Immunol. 2014;31:115-120.

52. Murakami-Satsutani N, et al. IL-33 promotes the induction and maintenance of $\mathrm{Th} 2 \mathrm{immune}$ responses by enhancing the function of OX 40 ligand. Allergol Int. 2014;63(3):443-455.

53. Turner DL, et al. Biased generation and in situ activation of lung tissue-resident memory CD4 T cells in the pathogenesis of allergic asthma. JImmunol. 2018;200(5):1561-1569.

54 . Dolence JJ, et al. Airway exposure initiates peanut allergy by involving the IL-1 pathway and T follicular helper cells in mice. J Allergy Clin Immunol. 2018;142(4):1144-1158.e8.

55. Gong F, et al. Circulating follicular T-helper cell subset distribution in patients with asthma. Allergy Asthma Proc. 2016;37(6):154-161.

56. Yao Y, Wang ZC, Yu D, Liu Z. Role of allergenspecific T-follicular helper cells in immunotherapy. Curr Opin Allergy Clin Immunol. 2018;18(6):495-501.

57. Frossard CP, Asigbetse KE, Burger D, Eigenmann PA. Gut T cell receptor- $\gamma \delta(+)$ intraepithelial lymphocytes are activated selectively by cholera toxin to break oral tolerance in mice. Clin Exp Immunol. 2015;180(1):118-130.

58. Bol-Schoenmakers M, et al. Regulation by intestinal $\gamma \delta \mathrm{T}$ cells during establishment of food allergic sensitization in mice. Allergy. 2011;66(3):331-340.

59. Peters C, Häsler R, Wesch D, Kabelitz D. Human V $22 \mathrm{~T}$ cells are a major source of interleukin-9. Proc Natl Acad Sci U S A. 2016;113(44):12520-12525.

60. Zheng R, Yang $\mathrm{Q}$. The role of the $\gamma \delta \mathrm{T}$ cell in allergic diseases. J Immunol Res. 2014;2014:963484.

61. Du Toit G, et al. Randomized trial of peanut consumption in infants at risk for peanut allergy. N Engl J Med. 2015;372(9):803-813.

62. Akdis CA, Akdis M. Mechanisms of immune tolerance to allergens: role of IL-10 and Tregs. J Clin Invest. 2014;124(11):4678-4680.

63. Berin MC, Shreffler WG. Mechanisms underlying induction of tolerance to foods. Immunol Allergy Clin North Am. 2016;36(1):87-102.

64. Kucuksezer UC, Ozdemir C, Akdis M, Akdis CA. Mechanisms of immune tolerance to allergens in children. Korean J Pediatr. 2013;56(12):505-513.

65. Rachid R, Umetsu DT. Immunological mechanisms for desensitization and tolerance in food allergy. Semin Immunopathol. 2012;34(5):689-702.

66. Sampath V, Tupa D, Graham MT, Chatila TA, Spergel JM, Nadeau KC. Deciphering the black box of food allergy mechanisms. Ann Allergy Asthma Immunol. 2017;118(1):21-27.

67. Tordesillas L, Berin MC. Mechanisms of oral tolerance. Clin Rev Allergy Immunol. 2018;55(2):107-117.

68. Yu W, Freeland DMH, Nadeau KC. Food allergy: immune mechanisms, diagnosis and immunotherapy. Nat Rev Immunol. 2016;16(12):751-765.

69. Bremel RD, Homan EJ. Extensive T-cell epitope repertoire sharing among human proteome, gastrointestinal microbiome, and pathogenic bacteria: implications for the definition of self. Front Immunol. 2015;6:538.

70. Lai L, et al. T cell repertoire following kidney transplantation revealed by high-throughput sequencing. Transpl Immunol. 2016;39:34-45.

71. Föhse L, et al. High TCR diversity ensures optima function and homeostasis of Foxp $3^{+}$regulatory $\mathrm{T}$ cells. Eur J Immunol. 2011;41(11):3101-3113.

72. Vickery BP, et al. AR101 oral immunotherapy for peanut allergy. $N$ Engl J Med . 2018;379(21):1991-2001.

73. Burks AW, et al. Sublingual immunotherapy for peanut allergy: long-term follow-up of a randomized multicenter trial. JAllergy Clin Immunol. 2015;135(5):1240-1248.e1.

74. Jones SM, et al. Epicutaneous immunotherapy for the treatment of peanut allergy in children and young adults. J Allergy Clin Immunol. 2017;139(4):1242-1252.e9.

75. Nelson HS, Lahr J, Rule R, Bock A, Leung D. Treatment of anaphylactic sensitivity to peanuts by immunotherapy with injections of aqueous peanut extract. J Allergy Clin Immunol. 1997;99(6 pt 1):744-751.

76. Lanser BJ, Leung DYM. The current state of epicutaneous immunotherapy for food allergy: a comprehensive review. Clin Rev Allergy Immunol. 2018;55(2):153-161.

77. Le UH, Burks AW. Oral and sublingual immunotherapy for food allergy. World Allergy Organ J. 2014;7(1):35.
78. Jones SM, et al. Clinical efficacy and immune regulation with peanut oral immunotherapy. JAllergy Clin Immunol. 2009;124(2):292-300.e1.

79. Varshney P, et al. A randomized controlled study of peanut oral immunotherapy: clinical desensitization and modulation of the allergic response. JAllergy Clin Immunol. 2011;127(3):654-660.

80. Bird JA, et al. Efficacy and safety of AR101 in oral immunotherapy for peanut allergy: results of ARC001, a randomized, double-blind, placebocontrolled phase 2 clinical trial. J Allergy Clin Immunol Pract. 2018;6(2):476-485.e3.

81. Hoeppli RE, Wu D, Cook L, Levings MK. The environment of regulatory $\mathrm{T}$ cell biology: cytokines, metabolites, and the microbiome. Front Immunol. 2015;6:61.

82. Pellerin L, et al. Peanut-specific Tr1 cells induced in vitro from allergic individuals are functionally impaired. J Allergy Clin Immunol. 2017;141(1):202-213.

83. Palomares F, et al. Immunological changes induced in peach allergy patients with systemic reactions by Pru $\mathrm{p} 3$ sublingual immunotherapy. Mol Nutr Food Res. 2018;62(3):1700669.

84. Sugimoto $\mathrm{M}$, et al. Differential response in allergen-specific IgE, IgGs, and IgA levels for predicting outcome of oral immunotherapy. Pediatr Allergy Immunol. 2016;27(3):276-282.

85. Meiler F, Klunker S, Zimmermann M, Akdis CA, Akdis M. Distinct regulation of IgE, IgG4 and IgA by $\mathrm{T}$ regulatory cells and toll-like receptors. Allergy. 2008;63(11):1455-1463.

86. James LK, Till SJ. Potential mechanisms for IgG4 inhibition of immediate hypersensitivity reactions. Curr Allergy Asthma Rep. 2016;16(3):23.

87. Ryan JF, et al. Successful immunotherapy induces previously unidentified allergen-specific $\mathrm{CD}^{+}{ }^{+} \mathrm{T}$-cell subsets. Proc Natl Acad Sci U S A. 2016;113(9):E1286-E1295.

88. Wambre E, et al. A phenotypically and functionally distinct human $\mathrm{TH} 2$ cell subpopulation is associated with allergic disorders. Sci Transl Med. 2017;9(401):eaam9171.

89. Chiang D, et al. Single-cell profiling of peanutresponsive $\mathrm{T}$ cells in patients with peanut allergy reveals heterogeneous effector $\mathrm{TH} 2$ subsets. J Allergy Clin Immunol. 2018;141(6):2107-2120.

90. Cianferoni A, et al. Invariant natural killer cells change after an oral allergy desensitization protocol for cow's milk. Clin Exp Allergy. 2017;47(11):1390-1397.

91. Srivastava KD, Qu C, Zhang T, Goldfarb J, Sampson HA, Li XM. Food Allergy Herbal Formula-2 silences peanut-induced anaphylaxis for a prolonged posttreatment period via IFN- $\gamma$-producing $\mathrm{CD} 8^{+} \mathrm{T}$ cells. J Allergy Clin Immunol. 2009;123(2):443-451

92. Han A, et al. Dietary gluten triggers concomitan activation of $\mathrm{CD}^{+}$and $\mathrm{CD} 8^{+} \alpha \beta \mathrm{T}$ cells and $\gamma \delta \mathrm{T}$ cells in celiac disease. Proc Natl Acad Sci US A. 2013;110(32):13073-13078.

93. Yu W, et al. Allergen specific CD $8^{+} \mathrm{T}$ cells in peanut allergic individuals [published online ahead of print January 22, 2019]. J Allergy Clin Immunol. https://doi.org/10.1016/j.jaci.2019.01.011.

94. Calzada D, Baos S, Cremades L, Cardaba B. New treatments for allergy: advances in peptide immunotherapy. Curr Med Chem. 
2018;25(19):2215-2232.

95. O'Hehir RE, Prickett SR, Rolland JM. T cell epitope peptide therapy for allergic diseases. Curr Allergy Asthma Rep. 2016;16(2):14.

96. Birrueta G, et al. Peanut-specific T cell responses in patients with different clinical reactivity. PLOS One. 2018;13(10):e0204620.

97. Renand A, et al. Heterogeneity of Ara h component-specific CD4 $\mathrm{T}$ cell responses in peanutallergic subjects. Front Immunol. 2018;9:1408.

98. Scheiblhofer S, Thalhamer J, Weiss R. DNA and mRNA vaccination against allergies. Pediatr Allergy Immunol. 2018;29(7):679-688.

99. Su Y, Connolly M, Marketon A, Heiland T. CryJ-LAMP DNA vaccines for Japanese red cedar allergy induce robust Th1-Type immune responses in murine model. JImmunol Res. 2016;2016:4857869.

100. Su Y, Romeu-Bonilla E, Anagnostou A, FitzPatrick D, Hearl W, Heiland T. Safety and longterm immunological effects of CryJ2-LAMP plasmid vaccine in Japanese red cedar atopic subjects: a phase I study. Hum Vaccin Immunother. 2017;13(12):2804-2813.

101. Lin C, et al. Combining anti-IgE with oral immunotherapy. Pediatr Allergy Immunol. 2017;28(7):619-627.

102. Andorf S, et al. Anti-IgE treatment with oral immunotherapy in multifood allergic participants: a double-blind, randomised, controlled trial. Lancet Gastroenterol Hepatol. 2018;3(2):85-94.

103. Abdel-Gadir A, et al. Oral immunotherapy with omalizumab reverses the Th2 cell-like pro- gramme of regulatory $\mathrm{T}$ cells and restores their function. Clin Exp Allergy. 2018;48(7):825-836.

104. Chang HY, Nadeau KC. IL-4R $\alpha$ inhibitor for atopic disease. Cell. 2017;170(2):222

105. Sampath V, Sindher SB, Zhang W, Nadeau KC. New treatment directions in food allergy. Ann Allergy Asthma Immunol. 2018;120(3):254-262.

106.Cukrowska B. Microbial and nutritional programming - the importance of the microbiome and early exposure to potential food allergens in the development of allergies. Nutrients. 2018;10(10):E1541.

107. Ho HE, Bunyavanich S. Role of the microbiome in food allergy. Curr Allergy Asthma Rep. 2018;18(4):27.

108. Arpaia N, et al. Metabolites produced by commensal bacteria promote peripheral regulatory $\mathrm{T}$-cel generation. Nature. 2013;504(7480):451-455.

109. Furusawa Y, et al. Commensal microbe-derived butyrate induces the differentiation of colonic regulatory T cells. Nature. 2013;504(7480):446-450.

110.Smith PM, et al. The microbial metabolites, short-chain fatty acids, regulate colonic Treg cell homeostasis. Science. 2013;341(6145):569-573.

111. Fu L, Peng J, Zhao S, Zhang Y, Su X, Wang Y. Lactic acid bacteria-specific induction of $\mathrm{CD} 4^{+} \mathrm{Foxp} 3$ $\mathrm{T}$ cells ameliorates shrimp tropomyosin-induced allergic response in mice via suppression of mTOR signaling. Sci Rep. 2017;7(1):1987.

112. Vonk MM, et al. Improved efficacy of oral immunotherapy using non-digestible oligosaccharides in a murine cow's milk allergy model: a potential role for Foxp $3^{+}$regulatory T cells. Front Immunol.
2017;8:1230.

113. Tsuda M, et al. Dietary fructo-oligosaccharides attenuate early activation of $\mathrm{CD} 4^{+} \mathrm{T}$ cells which produce both Th1 and Th2 cytokines in the intestinal lymphoid tissues of a murine food allergy model. Int Arch Allergy Immunol. 2017;174(3-4):121-132.

114. Tang ML, et al. Administration of a probiotic with peanut oral immunotherapy: a randomized trial. J Allergy Clin Immunol. 2015;135(3):737-744.e8.

115. Hsiao KC, Ponsonby AL, Axelrad C, Pitkin S, Tang MLK, PPOIT Study Team. Long-term clinical and immunological effects of probiotic and peanut oral immunotherapy after treatment cessation: 4-year follow-up of a randomised, double-blind, placebo-controlled trial. Lancet Child Adolesc Health. 2017;1(2):97-105.

116. Fu L, Song J, Wang C, Fu S, Wang Y. Bifidobacterium infantis potentially alleviates shrimp tropomyosin-induced allergy by tolerogenic dendritic cell-dependent induction of regulatory $\mathrm{T}$ cells and alterations in gut microbiota. Front Immunol. 2017;8:1536

117. Abarbanel DN, et al. Immunomodulatory effect of vancomycin on Treg in pediatric inflammatory bowel disease and primary sclerosing cholangitis. J Clin Immunol. 2013;33(2):397-406.

118. Josefowicz SZ, et al. Extrathymically generated regulatory $\mathrm{T}$ cells control mucosal TH2 inflammation. Nature. 2012;482(7385):395-399.

119. Angelo M, et al. Multiplexed ion beam imaging of human breast tumors. Nat Med. $2014 ; 20(4): 436-442$ 